\title{
Binary Alloys of Ge and Te: Order, Voids, and the Eutectic Composition
}

\author{
J. Akola ${ }^{1,2}$ and R. O. Jones ${ }^{1}$ \\ ${ }^{1}$ Institut für Festkörperforschung, Forschungszentrum Jülich, D-52425 Jülich, Germany \\ ${ }^{2}$ Nanoscience Center, Department of Physics, P.O. Box 35, FI-40014 University of Jyväskylä, Finland \\ (Received 10 January 2008; revised manuscript received 15 April 2008; published 23 May 2008)
}

\begin{abstract}
The liquid and amorphous structures of $\mathrm{Ge}_{0.15} \mathrm{Te}_{0.85}$ and $\mathrm{GeTe}$ alloys are characterized using combined density functional/molecular dynamics simulations. Te is threefold coordinated, in contrast with predictions of the " $8-N$ rule," and Ge atoms (fourfold coordinated) show octahedral and tetrahedral bonding angles. Cubic local environment occurs in both materials, and GeTe shows a pronounced alternation of atomic types. Tetrahedral Ge coordination is more common in the eutectic $\mathrm{Ge}_{0.15} \mathrm{Te}_{0.85}$, which comprises corner- and edge-sharing $\mathrm{GeTe}_{4}$ units surrounded by Te. There is no Te segregation, and the material resembles neither GeTe nor Te. The ubiquitous cavities (voids) have been overlooked in $\mathrm{Ge}_{0.15} \mathrm{Te}_{0.85}$, where they comprise over $25 \%$ of the volume.
\end{abstract}

DOI: 10.1103/PhysRevLett.100.205502

Alloys of $\mathrm{Ge}$ and $\mathrm{Te}\left(\mathrm{Ge}_{x} \mathrm{Te}_{1-x}\right)$, members of the large and important family of IV-VI alloys, have fascinated experimentalists and theorists for decades. They were the first to show real promise as phase change storage media [1], which are based on the rapid and reversible transition between crystalline $(c-)$ and amorphous $(a-)$ forms and play important roles in modern computers (DVD-RAM) and electronic devices (rewritable optical disks DVD-RW). Moreover, there are striking changes on melting, particularly near the eutectic composition $\mathrm{Ge}_{0.15} \mathrm{Te}_{0.85}$, where the density shows an anomalous increase with increasing temperature $T$, a semiconductor-to-metal transition occurs [2], and there are pronounced deviations from linearity in the sound velocity, adiabatic compressibility, and specific heat [3]. Many attempts to understand these phenomena have focused on structural changes, as we do here. We focus on the eutectic composition $(x=0.15)$, but insight into Terich systems $(x<0.5)$ can be found by a detailed comparison with GeTe $(x=0.5)$.

Scattering methods provide much less structural information about disordered phases than in crystals. Work on $\mathrm{Ge} / \mathrm{Te}$ alloys includes x-ray and neutron scattering [4-7], and extended $\mathrm{x}$-ray absorption fine structure [8-11] and Raman spectroscopies [8,9]. The reverse Monte Carlo method can be used to determine pair distribution functions from the measured structure factors $S(Q)$ [6,7], but the results can depend on the assumptions made. Structures of $a$-GeTe with Ge:Te coordinations of 3:3 and/or 4:2 have been proposed $[8,9,12]$, and the phase change in $\mathrm{GeTe}$ could be a tetrahedral-to-octahedral (umbrella) flip of $\mathrm{Ge}$ atoms [9] or a Peierls deformation in an octahedral environment $[4,5,7]$. It has been suggested that the structure of $a-\mathrm{Ge}_{0.15} \mathrm{Te}_{0.85}$ involves $\mathrm{GeTe}_{4}$ tetrahedra bridged by Te-Te bonds [6], and that the liquid near the melting point has regions with the short-range order of $\alpha$-GeTe and pure Te that are destroyed at higher $T$ [13].

Theory is challenged to provide new insights, and there have been recent studies of the electronic and geometrical
PACS numbers: 61.43.Bn, 61.43.Dq, 61.46. $-\mathrm{w}$, 71.15.Pd

structure of liquid $\mathrm{Ge}_{x} \mathrm{Te}_{1-x}$ alloys and the density anomaly mentioned above $[4,14-16]$. The density functional (DF) method has a well-established predictive value in many contexts, and its combination with molecular dynamics is ideal to study amorphous $(a-)$ and liquid $(\ell-)$ $\mathrm{Ge} / \mathrm{Te}$ alloys. The computational demands, however, have restricted simulations to liquids in small cells (usually 80 atoms or less) for some tens of picoseconds $[4,14,15]$. One clear prediction is the absence of tetrahedral order $\left(s p^{3}\right.$ hybridization) in $\ell-\mathrm{Ge}_{0.15} \mathrm{Te}_{0.85}$ [15]. Recently we reported DF calculations of the $\mathrm{Ge}_{2} \mathrm{Sb}_{2} \mathrm{Te}_{5}$ (GST) alloy with 460 atoms in the unit cell over hundreds of picoseconds [17]. Such times approach the experimental quenching time ( $\sim 1 \mathrm{~ns}$ ) and proved to be essential to describe the ordering correctly. We apply this approach here with similar simulation times to $a$ - and $\ell-\mathrm{Ge}_{0.15} \mathrm{Te}_{0.85}$ (15:85) with 216 atoms, and we compare the results with those for GeTe (50:50). Three factors are crucial: alternating fourmembered $A B A B$ rings (squares), tetrahedral Ge atoms, and, in particular, cavities ("voids").

We use the CPMD program [18] with the approximation of Perdew, Burke, and Ernzerhof [19] for the exchangecorrelation energy, Troullier-Martins pseudopotentials [20], and periodic boundary conditions with a single point $(\mathbf{k}=0)$ in the Brillouin zone. The kinetic energy cutoff of the plane wave basis is $20 \mathrm{Ry}$, and we use BornOppenheimer molecular dynamics with a Nosé-Hoover thermostat (frequency $800 \mathrm{~cm}^{-1}$, chain length 4) and time steps of 250 and 125 a.u. (6.050 and 3.025 fs) for initialization and data collection, respectively. The starting geometry was the (rocksalt) structure of $c$-GeTe, with 216 atoms in cubic cells with dimensions [18.61 $\AA$ (50:50), $19.70 \AA$ (15:85)] appropriate to the liquid and amorphous densities (0.0335 and 0.02827 atoms $/ \AA^{3}$, respectively) [21,22]. After heating to $5000 \mathrm{~K}$, GeTe was cooled (50 ps) to the melting point $(1000 \mathrm{~K})$, where data were collected (29 ps). After cooling to $300 \mathrm{~K}$ (115 ps) and data collection (26 ps), the temperature was lowered to $100 \mathrm{~K}$ 
TABLE I. Properties of $a$ - and $\ell-G e T e$ (see also Ref. [17]) and $\mathrm{Ge}_{0.15} \mathrm{Te}_{0.85}$. Simulations of $\ell-50: 50$ and $\ell-15: 85$ at 1000 and $680 \mathrm{~K}$, respectively. $r_{0}$, position of first maximum in RDF; $r_{\min }$, position of first minimum; $V_{c}$, total cavity volume; $\langle v\rangle$, average cavity size. For other symbols see text.

\begin{tabular}{lcccc}
\hline \hline & $a-50: 50$ & $\ell-50: 50$ & $a-15: 85$ & $\ell-15: 85$ \\
\hline$r_{0}(\AA)$ & 2.78 & 2.81 & 2.72 & 2.78 \\
$r_{\min }(\AA)$ & 3.68 & 3.87 & 3.37 & 3.46 \\
$n(\mathrm{Ge})$ & 4.2 & 4.5 & 4.0 & 3.7 \\
$n(\mathrm{Te})$ & 3.3 & 3.5 & 3.2 & 3.1 \\
$n_{\mathrm{Ge}-\mathrm{Te}}$ & 3.2 & 2.8 & 3.9 & 3.6 \\
$n_{\mathrm{Te}-\mathrm{Ge}}$ & 3.2 & 2.8 & 0.7 & 0.6 \\
$n_{\mathrm{Ge}-\mathrm{Ge}}$ & 1.1 & 1.7 & 0.1 & 0.2 \\
$n_{\mathrm{Te}-\mathrm{Te}}$ & 0.1 & 0.7 & 2.5 & 2.5 \\
$\alpha(\mathrm{Ge})$ & 0.56 & 0.32 & 0.97 & 0.94 \\
$\alpha(\mathrm{Te})$ & 0.94 & 0.55 & -0.09 & -0.11 \\
$V_{c}(\%)$ & 6.4 & 11.3 & 25.6 & 26.8 \\
$\langle\boldsymbol{v}\rangle\left(\AA^{3}\right)$ & 40.9 & 51.9 & 73.5 & 68.0 \\
$E_{g}(\mathrm{eV})$ & 0.13 & 0 & 0.10 & 0 \\
\hline \hline
\end{tabular}

(96 ps) and the structure optimized by simulated annealing. A similar procedure in $\mathrm{Ge}_{0.15} \mathrm{Te}_{0.85}$ provided data at $680 \mathrm{~K}$ (liquid, $47 \mathrm{ps}$ ) and $300 \mathrm{~K}$ (amorphous, $31 \mathrm{ps)}$ in a total simulation time of $300 \mathrm{ps}$. The electronic densities of states

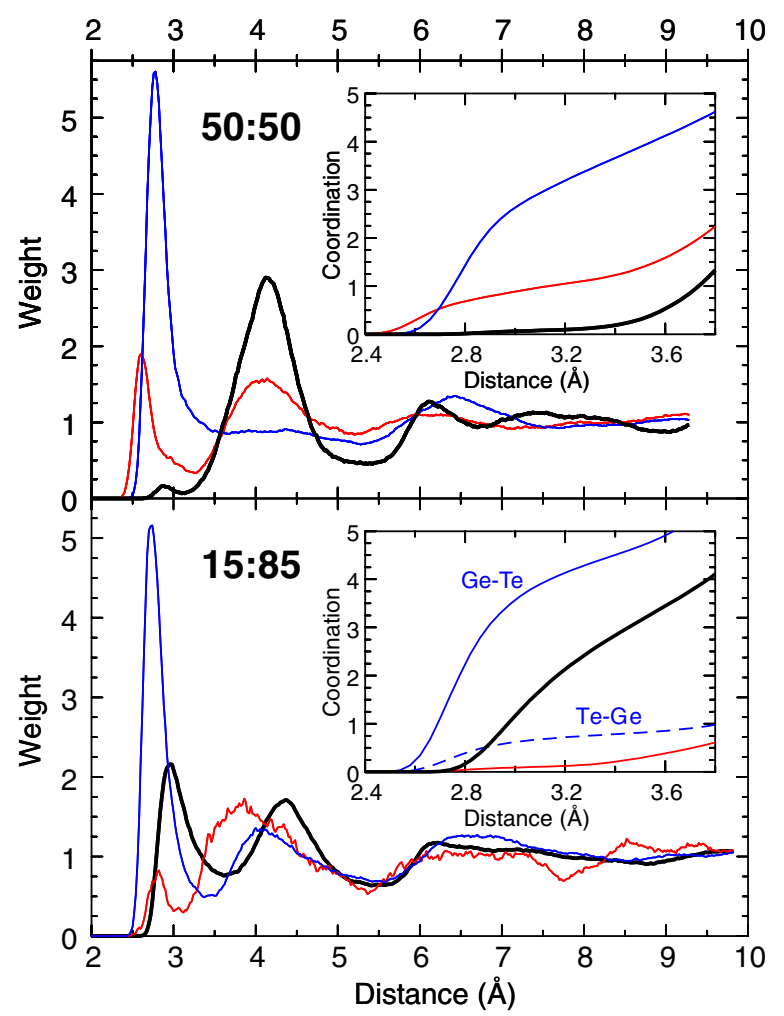

FIG. 1 (color online). Partial RDF of $a$-GeTe and $a-\mathrm{Ge}_{0.15} \mathrm{Te}_{0.85}$ at $300 \mathrm{~K}$. Black (thick) line, Te-Te; blue (peaked) line, $\mathrm{Ge}-\mathrm{Te}$ (Te-Ge); red (middle) line, Ge-Ge. Insets: Coordination numbers as a function of cutoff distance (here $3.2 \AA$ A).
TABLE II. Population analysis of fourfold coordinated Ge atoms in $a$ - and $\ell$-GeTe and $\mathrm{Ge}_{0.15} \mathrm{Te}_{0.85}$. The percentages of tetrahedrally coordinated $\mathrm{Ge}$ atoms are in parentheses, and the predominant contributions are in boldface.

\begin{tabular}{lcccc}
\hline \hline & $a-50: 50$ & $\ell-50: 50$ & $a-15: 85$ & $\ell-15: 85$ \\
\hline $\mathrm{Te}_{4}$ & $13.1(9.5)$ & $6.9(6.6)$ & $65.7(\mathbf{5 9 . 1})$ & $43.7(\mathbf{4 2 . 1})$ \\
$\mathrm{GeTe}_{3}$ & $32.3(19.2)$ & $15.9(13.0)$ & $6.6(2.4)$ & $7.9(5.7)$ \\
$\mathrm{Ge}_{3} \mathrm{Te}$ & 12.1 & 11.7 & & 0.3 \\
$\mathrm{Ge}_{3} \mathrm{Te}$ & 2.4 & 3.9 & & \\
Total & $59.9(28.7)$ & $38.9(19.6)$ & $72.3(\mathbf{6 1 . 5})$ & $51.9(\mathbf{4 7 . 8})$ \\
\hline \hline
\end{tabular}

and the band gaps $E_{g}$ (Table I, $64 \mathbf{k}$-point mesh) differ negligibly from the $\mathbf{k}=0$ values.

The partial radial distribution functions (RDF) and coordination numbers $n$ are shown in Fig. 1 and Table I. The long-range order of Te atoms in GeTe (long-range oscillations to $10 \AA$ ) was also found in GST (460 atoms) [17], but there is little sign of Te order in 15:85. Table I shows that $a$-GeTe has few Te-Te bonds, while Ge-Ge bonds are rare in $a-\mathrm{Ge}_{0.15} \mathrm{Te}_{0.85}$. Ge-Te bonds are shorter in 15:85 (Table I). GeTe contains numerous Ge-Ge bonds [the coordination number (1.1) is near the extended x-ray absorption fine structure value (1.2)], and $n(\mathrm{Ge})$ is slightly larger than the experimental value (3.7) [10]. The average coordination numbers are 4 for $\mathrm{Ge}$ and 3 for Te, and the most prominent configurations in $\mathrm{GeTe}$ are $\mathrm{Ge}-\mathrm{GeTe}_{3}$ (see Table II). $\mathrm{Ge}^{-\mathrm{Te}_{4}}$ are by far the most common in 15:85 [22].

The Ge-centered correlation maps for pairs of Ge-Te bonds show that $a-50: 50$ [Fig. 2(a)] has distances above $3.2 \AA$ that contribute to the broad first peak in the Ge-Te RDF (Fig. 1). These correlate mainly with Ge-Te bonds



FIG. 2 (color online). Distance-distance correlation maps of $\mathrm{Ge}-\mathrm{Te}$ bonds with respect to Ge atoms. GeTe at (a) $300 \mathrm{~K}$ and (b) $1000 \mathrm{~K} \cdot \mathrm{Ge}_{0.15} \mathrm{Te}_{0.85}$ at (c) $300 \mathrm{~K}$ and (d) $680 \mathrm{~K}$. 
near $2.8 \AA$, but there are Ge atoms with shorter bonds and less contribution from intermediate Ge-Te distances. Ge atoms can then be divided into tetrahedrally and octahedrally bonded [23], and the former dominate $\left(62 \%, s p^{3}\right.$ hybridized) in $a-15: 85$ [Table II, Fig. 2(c)]. This is validated by the nearest-neighbor and bond angle distributions [22]. In GeTe, however, most Ge atoms favor octahedral or cubic bond angles $\left(p^{3}\right)$, although sixfold coordination is found in only $3.4 \%$ or $11.0 \%$ of Ge atoms at 300 and $1000 \mathrm{~K}$, respectively. The liquids [Figs. 2(b) and 2(d)] show a broader range of Ge-Te bond pairs, and intermediate distances (bridges) are also evident in 15:85. Tetrahedral $\mathrm{GeTe}_{4}$ still dominate the statistics (Table II).

Order among Ge (type $A$ ) and Te (type $B$ ) atoms can be measured by the parameters $\alpha_{x}(x=\mathrm{Ge}$ or Te, Table I) [22], which depend on the concentration and coordination numbers of the components [17,24]. $\alpha_{x}$ ranges from -1 (segregation) to zero (random mixture) to +1 (perfect $A B$ ordering). Amorphous GeTe is "semiordered" $\left(\alpha_{x}>0.5\right.$, Table I), and even the liquid at $1000 \mathrm{~K}$ shows significant $A B$ alternation. Order tends to increase as simulations proceed, so the lower results of Ref. [4] $\left(\alpha_{\mathrm{Ge}}=0.17\right.$ and $\left.\alpha_{\mathrm{Te}}=0.32\right)$ may reflect the much shorter simulation times. The order parameters in 15:85 naturally reflect the large Te concentration.

The phase transition in GST can be viewed as the fusion of randomly oriented $A B A B$ squares $(A=\mathrm{Ge}, \mathrm{Sb})$ to form a rocksalt lattice [17]. Such squares are also essential in GeTe and are highlighted for $a$-GeTe in Fig. 3(a), where $75 \%$ of the atoms are involved. Even above the melting point $(1000 \mathrm{~K}), 41 \%$ of the atoms participate in $A B A B$ squares. Cubic order of Te and four-membered rings (not $A B A B$ ) are also favored in 15:85, and Fig. 3(b) shows a $\mathrm{Te}_{8}$ cube. The angular distribution of Te-Te-Te configurations is typical of octahedral structures, with a strong peak at $90^{\circ}$ and a weaker peak at $180^{\circ}$ [22]. The average coordination number of $\mathrm{Te}$ (3.2) differs from that expected for random covalent networks [25] ("8- $N$ rule") or the crystal structure of Te (parallel helical chains with bond angles of $\left.103.2^{\circ}\right)$.

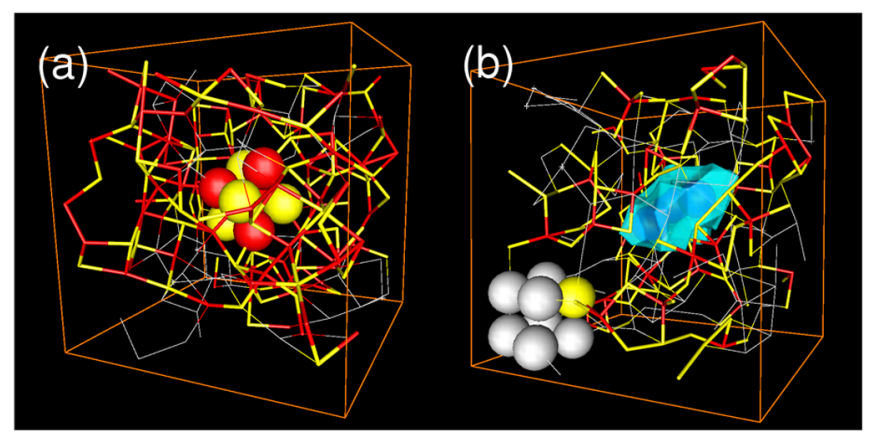

FIG. 3 (color online). (a) Simulation box of $a$-GeTe $(18.6 \AA$, 216 atoms) with $A B A B$ squares highlighted. An $A B A B$ cube is shown. Red, Ge; yellow, Te. (b) Simulation box of $\mathrm{Ge}_{0.15} \mathrm{Te}_{0.85}$ (19.7 $\AA, 216$ atoms) with $\mathrm{Ge}$ and Ge-coordinating Te atoms highlighted. A multivacancy and a cubic Te subunit are shown.
Cavities [22] are characteristic of $\mathrm{Ge}_{0.15} \mathrm{Te}_{0.85}$, occupying more than $25 \%$ of the total volume in both the amorphous and liquid states. Figure 4 shows their volume distributions, the vacancy-vacancy RDF (inset), and a typical medium-sized cavity. The volumes and shapes vary widely (see Table I), and the RDF show the presence of both multivacancies and long-range order in the amorphous state. The cavity distributions are significantly $T$ dependent: there are more in the liquid $(680 \mathrm{~K})$ and more that are smaller, whereas larger cavities $\left(70-120 \AA^{3}\right)$ are more abundant at $300 \mathrm{~K}$. The complicated shapes of the latter reflect the enhanced cubic local order of Te in the amorphous phase. The tails in Fig. 3(b) correspond to diand multivacancies and are almost identical in the two phases. In GeTe, whose crystalline structure is vacancyfree, cavities comprise $6.4 \%$ of the volume of the amorphous form. These cavities are surrounded mainly by Te atoms and provide the space needed to reorient $A B A B$ squares in the $a$ to $c$ transition [17]. The larger cavity volume $V_{c}$ in the liquid $(1000 \mathrm{~K})$ reflects the larger average coordination number of the atoms.

Three types of Te atom occur near the eutectic composition: (a) atoms bonded to Ge [first coordination shell, highlighted in Fig. 3(b)], (b) atoms in the second coordination shell bonded to (a)-type atoms, and (c) atoms inside Te domains in a matrix of Ge-centered units (mostly $\left.\mathrm{GeTe}_{4}\right) . \mathrm{In} \mathrm{Ge}_{0.15} \mathrm{Te}_{0.85}, 45 \%, 31 \%$, and $9 \%$ of all atoms are of types (a), (b), and (c), respectively, so that three (a)type Te atoms occur for each Ge atom. Most domains of type (c) contain a single atom; i.e., the system with $85 \% \mathrm{Te}$

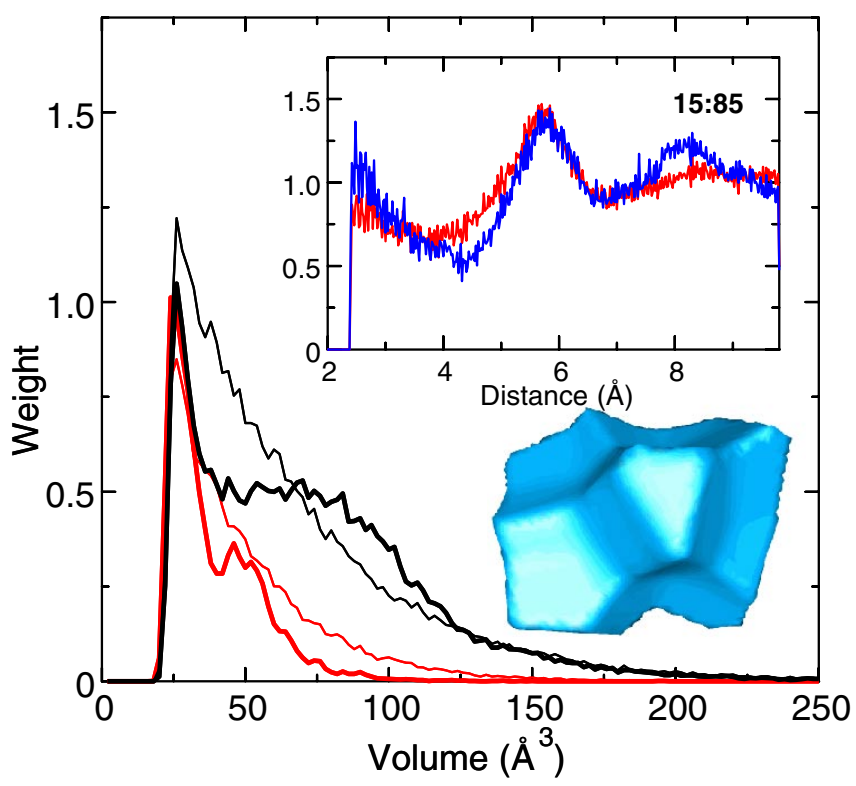

FIG. 4 (color online). Volume distribution of cavities and a medium-sized cavity $\left(99.3 \AA^{3}\right.$ ). Black, $\mathrm{Ge}_{0.15} \mathrm{Te}_{0.85}$ at $300 \mathrm{~K}$ (amorphous, thick line) and $680 \mathrm{~K}$ (liquid, thin line); red, GeTe at $300 \mathrm{~K}$ (thick line) and $1000 \mathrm{~K}$ (thin line). Inset: vacancyvacancy $\mathrm{RDF}$ of $\mathrm{Ge}_{0.15} \mathrm{Te}_{0.85}$ at $300 \mathrm{~K}$ (blue/black, larger oscillations) and $680 \mathrm{~K}$ (red/gray). 
shows no significant Te segregation. To estimate the rapid increase in segregation that must occur at higher Te concentrations, we note that - in a material comprising $\mathrm{GeTe}_{4}$ units - the average Ge atom shares two Te with neighboring $\mathrm{Ge}$ (corner- or edge-sharing tetrahedra). If the ratio between the number of (a)- and (b)-type Te atoms is assumed to be constant, then $40 \%$ and $70 \%$ of the Te atoms are of type (c) in the 10:90 and 5:95 alloys, respectively.

The results provide information for a range of $\mathrm{Ge} / \mathrm{Te}$ alloys and help us to understand the special nature of the eutectic 15:85, whose main structural feature can be viewed as an array of (tetrahedral) $\mathrm{GeTe}_{4}$ units connected by (b)-type Te atoms (see Table II). This picture of $\ell-15: 85$ is quite different from that of Ref. [15] (8 Ge and $48 \mathrm{Te}$ atoms), where tetrahedrally coordinated Ge atoms play no role at $680 \mathrm{~K} . A B A B$ squares are essential units in $c$ - and $a$-GeTe, but their fraction falls dramatically (by $\sim 90 \%$ ) as the Te concentration increases to $15: 85$, and the small number of (c)-type Te atoms must increase rapidly for $\mathrm{Te}$ concentrations above $85 \%$. The eutectic composition occurs then in the small region where neither $A B A B$ squares nor Te domains are common. Our analysis suggests that the density anomaly is associated with the large vacancy volume and the lack of cubic local order of Te atoms in the melt. We have performed other calculations that indicate that this applies to Te and other Te-rich alloys.

The calculated atomic mean-square displacements of both liquid alloys show a linear dependence on time, and the diffusion constants are $D_{\mathrm{Ge}}=4.65 \times 10^{-5}$ and $D_{\mathrm{Te}}=$ $3.93 \times 10^{-5} \mathrm{~cm}^{2} \mathrm{~s}^{-1}$ for $50: 50$ at $1000 \mathrm{~K}$, and $D_{\mathrm{Ge}}=$ $2.46 \times 10^{-5}$ and $D_{\mathrm{Te}}=2.89 \times 10^{-5} \mathrm{~cm}^{2} \mathrm{~s}^{-1}$ for $15: 85$ at $680 \mathrm{~K}$. The lower mobility of Ge in 15:85 correlates well with the abundance of tetrahedral Ge-Te $\mathrm{T}_{4}$ units in the melt (Table II). Viscosity estimates depend on the particle radius in the Stokes-Einstein relation, and a plausible value $(1.4 \AA)$ leads to 1.2 and $1.3 \mathrm{cP}$ for $50: 50$ and $15: 85$, respectively. The measured value of $\ell$-GeTe ranges from 1.9 and $1.2 \mathrm{cP}$ between 1000 and $1200 \mathrm{~K}$ [26].

The structures of the amorphous and liquid phases of GeTe and the eutectic $\mathrm{Ge}_{0.15} \mathrm{Te}_{0.85}$ have been characterized using DF simulations (216 atoms for hundreds of picoseconds). GeTe prefers octahedral bond angles and $A B A B$ squares (strong $A B$ alternation), whereas corner- and edgesharing $\mathrm{Ge}^{-\mathrm{Te}_{4}}$ tetrahedra are common in 15:85. The threefold (cubic) coordination of Te found in both materials deviates from the " $8-N$ rule," where $N$ is the number of valence electrons (6). Cavities are always present, especially in $\mathrm{Ge}_{0.15} \mathrm{Te}_{0.85}(26 \%-27 \%$ of total volume), which resembles neither $\mathrm{GeTe}(A B A B$ squares) nor pure $\mathrm{Te}(\mathrm{Te}$ segregation). The small concentration of $\mathrm{Ge}$ and the large cavities in $\mathrm{Ge}_{0.15} \mathrm{Te}_{0.85}$ indicate the value of even larger simulations, and these are planned.

The calculations were performed on IBM Blue Gene/L and p690 computers in the FZ Jülich with grants from the FZJ and the John von Neumann Institute for Computing
(NIC). We thank I. Kaban, T. Matsunaga, and Y. Tsuchiya for providing original data.

[1] M. Chen, K. A. Rubin, and R. W. Barton, Appl. Phys. Lett. 49, 502 (1986).

[2] Y. Tsuchiya and H. Saitoh, J. Phys. Soc. Jpn. 62, 1272 (1993).

[3] Y. Tsuchiya, J. Non-Cryst. Solids 312-314, 212 (2002).

[4] J. Y. Raty et al., Phys. Rev. B 65, 115205 (2002).

[5] C. Bergman et al., Phys. Rev. B 67, 104202 (2003).

[6] P. Jóvári et al., J. Phys. Condens. Matter 17, 1529 (2005).

[7] M.-V. Coulet et al., Phys. Rev. B 72, 174209 (2005).

[8] A. V. Kolobov et al., J. Phys. Condens. Matter 16, S5103 (2004).

[9] K.S. Andrikopoulos et al., J. Phys. Condens. Matter 18, 965 (2006).

[10] Y. Maeda and M. Wakagi, Jpn. J. Appl. Phys. 30, 101 (1991).

[11] K. Hirota, K. Nagino, and G. Ohbayashi, J. Appl. Phys. 82, 65 (1997).

[12] S. J. Pickard, Y. P. Sharma, and J. P. de Neufville, J. NonCryst. Solids 34, 183 (1979).

[13] H. Neumann et al., J. Non-Cryst. Solids 97\&98, 1251 (1987).

[14] G. Zhao et al., Phys. Rev. B 74, 184202 (2006).

[15] C. Bichara, M. Johnson, and J. Y. Raty, Phys. Rev. Lett. 95, 267801 (2005).

[16] A. H. Edwards et al., Phys. Rev. B 73, 045210 (2006).

[17] J. Akola and R. O. Jones, Phys. Rev. B 76, 235201 (2007).

[18] CPMD V3.11: copyright IBM Corporation 1990-2006; copyright MPI für Festkörperforschung Stuttgart 19972001 (http://www.cpmd.org).

[19] J. P. Perdew, K. Burke, and M. Ernzerhof, Phys. Rev. Lett. 77, 3865 (1996).

[20] N. Troullier and J. L. Martins, Phys. Rev. B 43, 1993 (1991). The valence configurations are $4 s^{2} 4 p^{2}(\mathrm{Ge})$ and $5 s^{2} 5 p^{4}(\mathrm{Te})$, and the scalar-relativistic pseudopotentials contain nonlinear core corrections.

[21] The measured density of $a-15: 85$ (0.0282 atoms $/ \AA^{3}[\mathrm{~S}$. Kohara and T. Usuki (private communication)] is almost identical to the liquid value (0.02827).

[22] See EPAPS Document No. E-PRLTAO-100-043821 for references on the densities, definitions of cavities and order parameters $\alpha$, structure factors of $\ell-\mathrm{GeTe}$ and $\ell-\mathrm{Ge}_{0.15} \mathrm{Te}_{0.85}$, and the local structure of $a-\mathrm{Ge}_{0.15} \mathrm{Te}_{0.85}$. For more information on EPAPS, see http://www.aip.org/ pubservs/epaps.html.

[23] Ge atoms are denoted "tetrahedral" if the average deviation of the six bond angles from the octahedral values $\left(90^{\circ}, 180^{\circ}\right)$ exceeds $10^{\circ}$.

[24] H. Ruppersberg and C. N. J. Wagner, J. Non-Cryst. Solids 55, 165 (1983).

[25] F. Betts, A. Bienenstock, and S. R. Ovshinsky, J. NonCryst. Solids 4, 554 (1970).

[26] V.M. Glazov, S. N. Chizhevskaya, and N. N. Glagoleva, Liquid Semiconductors (Plenum, New York, 1969). 